\title{
Medication Safety in Hospital
}

\author{
*Ibrahim Dughaim Alreshidi \\ Department of Pharmacy, Maternity EChildren's Hospital, Hafr Albatin, Kingdom of Saudi Arabia
}

\begin{abstract}
The World Health Organization has recommended the adoption of basic applications in healthcare facilities to enhance medication safety over the years, but it remains unclear if these recommendations are adhered to by hospitals. We assessed the availability of primary medication safety practices in Saudi Arabian hospitals. Survey were carried out in 70 hospitals to identify the presence of core medication safety practices in Saudi Arabian hospitals and revealed that there were room and opportunity for improvement, even for cheap interventions. According to the study, only about $29 \%$ of the Saudi Arabian hospitals had a proper functioning drug safety committee, and $10 \%$ had a designated medication safety officer. It was also revealed that only $32 \%$ of the hospitals had the list of LASA authorized drugs, and $51 \%$ had a list of error-prone abbreviations and medical terms. Saturated electrolytes were also found as floor stock in $60 \%$ of all hospitals.
\end{abstract}

Key Words: Medication safety, Hospitals, Adverse drug event (ADEs), Look alike sound alike (LASA).

\section{INTRODUCTION}

"To Err is Human", however, in healthcare profession this could lead to serious consequences and in severe cases, can lead to loss of lives also. As we are getting civilized, more and more people are getting admitted into the hospitals and reportable incidents of medication errors are also rising. Instead of denying this fact, many developed countries have stepped forward and published their "mistakes" so that other people can take lesson from them. However, very few studies have been conducted in Saudi Arabia to report the actual situation. Although, medication safety is a universal concern among hospitals and medical experts, but these safety issues that affect the future of medication safety in Saudi Arabia have not been addressed well so far. This study will discuss the perspectives of medical practitioners on today issues about medication safety in hospitals and community health facilities in Saudi Arabia to identify issues to enhancing it and discuss the future of medication safety regulations and practices.

\section{METHODS}

\section{Medication Safety}

Even though Medication errors are common they are preventable; adverse drug events are a major cause of harm. For example, in the USA, one study found that $7 \%$ of adult patients in most hospitals experienced ADEs, and $27 \%$ of these ADEs were preventable and occurred due to negligence. In Saudi Arabia, there is a need to educate and provide information to healthcare experts regarding the importance of drug safety since most of the studies conducted in Saudi Arabia concluded that there is a deficit of culture of drug safety in Saudi Arabia among all levels of healthcare experts. According to Alanazi et al. (2016), it has been reported that $35 \%$ of medications dispensed to patients without proper prescription in Saudi Arabia are prescription. Also, it was found that $37 \%$ of patients admitted to the hospital had a medication discrepancy at the time
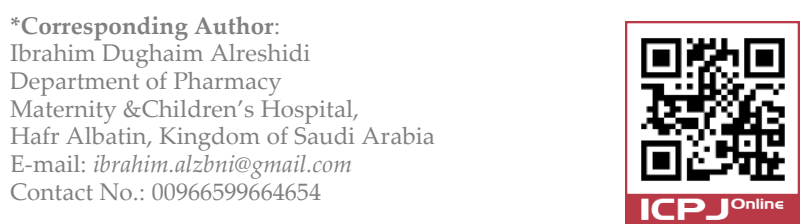

of hospital admission; this could have been prevented by an accurate reconciliation of medications.

\section{Patient Safety}

Most of the side effects are predictable and are clearly inscribed in the leaflets for each dose/drug. The primary problem is that some of the drugs' side effects were not previously documented or experienced, and the main risk here is if they would make patients experience severe effects when they are using them. The factors that may enhance the severity of the side effects, the type of medications and the age of the patients using them are the most critical. Some of these drugs might not cause any serious complications; examples are some types of antibiotics. On the other hand, some medications may cause serious side effects; examples include certain cancer treatment drugs, diabetic medications, and blood lipids treatment. Aljadhey et al. (2016) added that serious adverse reactions arise from commonly used and well-known treatment. Such as most patients believe that paracetamol, normally sold as Panadol is very safe and if taken it will not cause any severe side effects. However, paracetamol can cause serious side effects if consumed in high doses, particularly to the liver. The fact that makes this drug dangerous is that it is present in many combinations used to treat cold and flu, and most patients might not have this knowledge and take extra doses, leading to direct liver destructions (Al-Jeraisy, M. I., Alanazi, M. Q., \& Abolfotouh, M. A).

Up until now, there are drugs in Saudi Arabia that should be withdrawn from the market due to safety challenges concerning their relation to the emergence of severe side effects. For example, the recent withdrawal of the antidiabetic drug, Avandia, from Saudi Arabia and the global market in general as a result of its relationship with the occurrence of severe heart attacks. Therefore, Alanazi et al. (2016) found the idea of patient and drug safety a very critical and comprehensive idea that is considered a priority concerning the use of medications. Moreover, the responses to medication are different between males and females due to the physiological differences brought about by genders. However, there are patient groups that need close supervision and extra care when using medications, such as pregnant women, the elderly, and children. 
Table 1: Medication safety practices in Saudi Arabia hospitals.

\begin{tabular}{|c|c|}
\hline Factor & $\begin{array}{c}{ }^{* *} \text { Number of Hospitals } \\
\text { N (\%) }\end{array}$ \\
\hline \multicolumn{2}{|l|}{ Medication Safety Committee and error reporting systems } \\
\hline Medication safety committee & $22(28)$ \\
\hline Medication safety director & $7(9)$ \\
\hline Paper-based error reporting system used & $59(76)$ \\
\hline Electronic error reporting system implemented & $6(12)$ \\
\hline \multicolumn{2}{|l|}{ Look-Alike sound-Alike (LASA) medications } \\
\hline List (LASA) medications & $26(33)$ \\
\hline Mechanism for reviewing LASA medications & $20(47)$ \\
\hline Mechanism to prevent LASA medications & $35(57)$ \\
\hline Education on LASA medications & $38(50)$ \\
\hline Medications stored in pharmacy alphabetically & $57(73)$ \\
\hline Diagnosis field exists in the prescription or drug order & $73(95)$ \\
\hline Both brand and generic names included on medication labels & $20(27)$ \\
\hline \multicolumn{2}{|l|}{ Control of concentrated electrolyte solution } \\
\hline Concentrated electrolytes found on floor stock & $47(60)$ \\
\hline Second person verifies final concentrations of parenteral electrolyte solutions including calculations & $39(53)$ \\
\hline High-risk warning label used on diluted electrolyte solution & $26(34)$ \\
\hline \multicolumn{2}{|l|}{ Transition in care } \\
\hline New order required with patient admission or transfer & $46(59)$ \\
\hline Orders "resume the same medications" are accepted & $44(56)$ \\
\hline Policy to update medication list exists & $52(70)$ \\
\hline Complete drug history taken & $71(95)$ \\
\hline Pharmacist takes medication history & 0 \\
\hline Current medications list put in consistent highly visible location & $61(81)$ \\
\hline Written policies and procedures to list and update the medication list & $41(57)$ \\
\hline Current medication list updated with new physician orders & $62(83)$ \\
\hline List of discharge medications & $27(37)$ \\
\hline Health care professionals educated on procedures for reconciling medications & $18(24)$ \\
\hline \multicolumn{2}{|l|}{ Information Technology } \\
\hline Electronic access to inpatient laboratory values & $34(44)$ \\
\hline Medication bar coding & $9(12)$ \\
\hline Electronic medication administration record & $21(29)$ \\
\hline Pharmacy uses computer to enter prescription & $45(61)$ \\
\hline Patient allergy history is required to enter an order & $13(39)$ \\
\hline Drug allergy verified & $24(55)$ \\
\hline Pharmacy computer screens drug for drug allergy & $13(29)$ \\
\hline Allergy list is clearly visible on all pages of medication administration records & $53(77)$ \\
\hline Computer is directly interfaced with the laboratory & $10(14)$ \\
\hline Body weight is a required field & $8(11)$ \\
\hline \multicolumn{2}{|l|}{ Drug Information } \\
\hline Drug information resources in all patient care areas & $47(61)$ \\
\hline Computerized drug information resources in the pharmacy & $33(43)$ \\
\hline \multicolumn{2}{|l|}{ Other Medication Safety Practices } \\
\hline Renal or hepatic dosage adjustment for relevant patients & $18(24)$ \\
\hline Maximum dose for high alert drug & $20(27)$ \\
\hline Controlled drug formulary system & $57(75)$ \\
\hline A list of error prone abbreviations is available & $38(50)$ \\
\hline Unit dose system implemented & $70(93)$ \\
\hline Medications brought from home by patient are not used & $60(83)$ \\
\hline Discontinued medications are removed from patient supplies in a timely manner & $62(86)$ \\
\hline Pharmacy staff receive baseline competency evaluation & $42(56)$ \\
\hline
\end{tabular}

\footnotetext{
${ }^{* *}$ Total number of hospitals $=70$.
} 
during all the trimesters without consulting medical experts about the safety of the medication as it may have an impact on the formation of the fetus. Aljadhey, Mahmoud et.al) insisted that the danger of impact of drugs is not confined to taking drugs during the pregnancy period but severe effects may occur to the fetus. It may happen even if the medication is taken within several weeks before the pregnancy. In case of the children, it is important to exercise extreme caution when administering any medications for children since most of these medications are grouped depending on age group - some drugs are administered to children between 12-18 years only and also some are for use in children less than two years old. The main reasons are that most of this medication lack the scientific research and clinical trials required to examine the safety of these drugs in each age group separately. Vuong \& Marriott (2006) added that, children's vital organs are not developed or fully grown therefore the exposure to certain medications may lead to toxic side effects as the body is unable to metabolize the medication fully. These drugs increase the presence of active compounds in the body for a prolonged period and increase in their levels in the body, producing toxic effects. For senior citizens, this geriatric group is categorized as the most vulnerable to the impact of medications and so it is imperative to confirm their health status before prescribing any medication. The physiological functions of most body organs of old people decline with age, especially crucial organs such as the liver and kidneys. Communication is a key aspect of medical practice, both among healthcare experts and between healthcare officers and patients. In the recent study in Saudi Arabia hospitals showed that behavior and communication incidents among medical staff were one of the major incidents reported through a voluntary incident reporting system.

\section{RECOMMENDATION}

The above interventions can be implemented with an increase in resources allocated, and additional improvement in drug safety may be experienced with the implementation of solutions such as computer order entry and barcoding, and the adoption of the lists of LASA medications, lists of discharge medications and lists of prohibited abbreviations. Another solution is the removal of concentrated electrolyte solutions from floor stock and the Installation of state-of-the-art applications in a hospital that will help the medical experts to keep the record of patients' medical history (Walston, S. L., Al-Omar, B. A., \& Al-Mutari, F. A). the hospitals should evaluate innovative interventions, including their cost effectiveness, embark on teaching the public on matters involving medication safety and help in implementing applications to improve the safe use of medications. Continuous education and regular competency evaluation based on medication safety are required to enhance the current practice in Saudi Arabia and help train healthcare experts about best practices in medication safety. There is also Need for national initiatives and support from the national accreditation body to help restore and maintain medication safety practices in hospitals. Alahmadi suggested that the medical bodies and national initiatives are also required to promote a safety culture in the Saudi Arabian hospitals.

\section{CONCLUSION}

The study has discussed how several issues such as unrestricted public access to medication, communication gaps, and the lack of rigorous medication safety drills and methodologies in hospitals and the limited or no use of important medical technologies have contributed to the medical accidents across Saudi Arabian hospitals. Drug safety has undergone through various stages from the last decade, and there have been several accidents that hospitals should learn from to protect their patients. The study also discusses how all patients should be protected and specific groups of patients such as pregnant women, children and seniors should be given extra care and attention, since they are categorized as vulnerable populations. The paper also discusses how drugs can react with other drugs or foods used on a daily basis, and special precautions are needed to prevent these dangerous interactions. The study discusses how the risks from medications could be minimized in Saudi Arabian hospitals through patient education about drug safety and openness with the patient, which involves allowing them to ask for clarification related to their disease and medications. A healthy relationship between the medical experts and the patient is one of the most important determinants for drug safety that can be applied in Saudi Arabian hospitals.

\section{REFERENCES}

Al Hamid A, Aslanpour Z, Aljadhey H, Ghaleb M. (2016) Hospitalization Resulting from Medicine-Related Problems in Adult Patients with Cardiovascular Diseases and Diabetes in the United Kingdom and Saudi Arabia. International journal of environmental research and public health. 9; 13(5):479. [DOI]

Alahmadi, H. A. (2010). Assessment of patient safety culture in Saudi Arabian hospitals. Quality and Safety in Health Care, 19(5), 1-5. [DOI]

Alanazi, M. A., Tully, M. P. \& Lewis, P. J. (2016) A systematic review of the prevalence and incidence of prescribing errors with high-risk medicines in hospitals. Journal of Clinical Pharmacy and Therapeutics, 41 (3), pp. 239-245. [DOI]

Aljadhey H, Alhossan A, Alburikan K, Adam M, Murray MD, Bates DW. (2016a) Corrigendum to "Medication safety practices in hospitals: A national survey in Saudi Arabia" [Saudi P harm. J. 21 (2)(2013) 159-164]. Saudi Pharmaceutical Journal: SPJ. 24(2):232. [DOI]

Aljadhey H, Mahmoud MA, Ahmed Y, Sultana R, Zouein S, Alshanawani S, Mayet A, Alshaikh MK, Kalagi N, Al Tawil E, El Kinge AR. (2016b) Incidence of adverse drug events in public and private hospitals in Riyadh, Saudi Arabia: the (ADESA) prospective cohort study. BMJ open. 6(7):e010831. [DOI]

Al-Jeraisy, M. I., Alanazi, M. Q. \& Abolfotouh, M. A. (2011). Medication prescribing errors in a pediatric inpatient tertiary care setting in Saudi Arabia. BMC Research Notes, 4: 294. [DOI]

Vuong, T. \& Marriott, J. L. (2006) Unnecessary Medicines Stored in Homes of Patients at Risk of Medication Misadventure. Journal of Pharmacy Practice and Research, 36(1), pp. 16-20. [DOI]

Walston, S. L., Al-Omar, B. A., \& Al-Mutari, F. A. (2010). Factors affecting the climate of hospital patient safety: A study of hospitals in Saudi Arabia. International journal of health care quality assurance, 23(1), 35-50. [DOI] 